\title{
FEASIBILITY STUDY OF AN EXPERIMENT TO MEASURE THE VACUUM MAGNETIC BIREFRINGENCE
}

\author{
L. Duvillaret ${ }^{1}$, M. Finger ${ }^{2}$, M. Kral ${ }^{3}$, P. Pugnat ${ }^{3}$, A. Siemko ${ }^{3}$, J. Zicha ${ }^{4}$
}

The use of a recently decommissioned 15-meters long twin aperture LHC superconducting magnet prototype having a transverse magnetic field $\mathrm{B} \sim 9.5 \mathrm{~T}$ provides the unique opportunity for the construction of a new powerful experiment to measure the Vacuum Magnetic Birefringence (VMB). The values or the limit values of the mass and of the coupling constant to two photons of possible dark matter candidates such as axions are aimed to be deduced from such an experiment. In this article, the technical feasibility study of a new setup to measure the VMB will be presented. It is based on a linear optical resonant cavity housed in the LHC superconducting dipole prototype. The mechanical integrations of the optical components inside the magnet aperture as well as the optical detection principles will be presented. A comparison of the expected performances with respect to the present reference results for this type of experiment will also be given.

1 LAHC, Université de Savoie, France

2 Charles University, Faculty of Mathematics and Physics, Prague, Czech Republic

3 CERN, Accelerator Technology Department, Geneva, Switzerland

4 Technical University, Faculty of Mechanical Engineering, Prague, Czech Republic

Presented at the "Symmetries and Spin" Conference 2004

5-10 July 2004, Prague, Czech Republic

To be published in Czechoslovak Journal of Physics, Vol. 55 (2005)

Administrative Secretariat

AT Department

CERN

CH - 1211 Geneva 23
Geneva, Switzerland

30 June 2005 


\title{
Feasibility study of an experiment to measure the Vacuum Magnetic Birefringence
}

\author{
Pierre Pugnat, Miroslav Kral, Andrzej Siemko \\ CERN, Accelerator Technology Department, Geneva, Switzerland \\ Lionel Duvillaret \\ LAHC, Université de Savoie \\ Miroslav Finger \\ Charles University, Faculty of Mathematics and Physics, Prague, Czech Republic \\ Josef ZiCHA \\ Technical University, Faculty of Mechanical Engineering, Prague, Czech Republic
}

\begin{abstract}
The use of a recently decommissioned 15-meters long twin aperture LHC superconducting magnet prototype having a transverse magnetic field $\mathcal{B} \sim 9.5 T$ provides the unique opportunity for the construction of a new powerful experiment to measure the Vacuum Magnetic Birefringence (VMB). The values or the limit values of the mass and of the coupling constant to two photons of possible dark matter candidates such as axions are aimed to be deduced from such an experiment. In this article, the technical feasibility study of a new setup to measure the VMB will be presented. It is based on a linear optical resonant cavity housed in the LHC superconducting dipole prototype. The mechanical integrations of the optical components inside the magnet aperture as well as the optical detection principles will be presented. A comparison of the expected performances with respect to the present reference results for this type of experiment will also be given.
\end{abstract}

PACS: $14.80 \mathrm{Gt}, 12.20 . \mathrm{Fv}, 14.80 . \mathrm{Am}$

Key words: Vacuum magnetic birefringence, Axions, QED, QCD

\section{Introduction}

Since its prediction in 1936 by Euler and Heisenberg [1] and independently by Weisskopf [2] in the earlier development of the Quantum Electrodynamic (QED) theory, the Vacuum Magnetic Birefringence (VMB) is still a challenge for optical metrology techniques. According to the QED theory [3], the vacuum behaves as an optically active medium in the presence of an external magnetic field. It can be experimentally studied by using as a probe, a linearly polarised laser light beam [4]. After propagating through the vacuum submitted to a transverse magnetic field, the polarisation of the laser beam will change to elliptical and the parameters of the polarisation are directly related to fundamental constants such as the fine structure constant and the electron Compton wavelength. Contributions to the VMB could also arise from the existence of light scalar or pseudo-scalar particles like axions that couple to two photons and this would manifest itself as a sizeable deviation from the pure QED prediction [5]. 


\section{Theoretical Background}

\subsection{The pure QED prediction}

The pioneering works of Euler, Heisenberg [1], and Weisskopf [2] had shown that the classical electromagnetism theory must be corrected by nonlinear terms due to the probability of pair creation i.e. the vacuum polarisation effects. This is generally expressed by some corrective terms in the classical expression of the Lagrangian density $\mathcal{L}_{0}=\epsilon_{0}\left(\mathcal{E}^{2}-c^{2} \mathcal{B}^{2}\right) / 2$ of the vacuum submitted to an electrical field $\mathcal{E}$ and a magnetic field $\mathcal{B}$. Omitting terms of order of $\alpha^{3}$, the Euler-Heisenberg-Weisskopf (EHW) effective Lagrangian density can be written as:

$$
\mathcal{L}_{E H W}=\epsilon_{0} \frac{\left(\mathcal{E}^{2}-c^{2} \mathcal{B}^{2}\right)}{2}+\frac{2 \epsilon_{0}^{2} \lambda_{e}^{3} \alpha^{2}}{45 m_{e} c^{2}}\left[\left(\mathcal{E}^{2}-c^{2} \mathcal{B}^{2}\right)^{2}+7 c^{2}(\mathcal{E} \cdot \mathcal{B})^{2}\right]
$$

where $\epsilon_{0}$ is the linear permittivity of the vacuum, $\alpha$ is the fine structure constant, $t_{e}$ is the electron Compton wavelength, $m_{e}$ is the electron mass and $c$ is the speed of the light. The expression (1) can be considered as the first terms of a development in power of the only two Lorentz- and gauge-invariant combinations of second order in the fields containing no derivatives. For the case which is considered here, it can be shown that the EHW terms are much more important than the corrections that involve field derivatives [6].

From the derivatives of the EHW effective Lagrangian density with respect to $\mathcal{E}$ and $\mathcal{B}$, the components of the electric and magnetic relative permeability tensors of the vacuum can be deduced:

$$
\begin{array}{r}
\epsilon_{i j} \approx \delta_{i j}+\frac{4 \lambda_{e}^{3} \alpha^{2}}{45 m_{e} c^{2}}\left[2\left(\mathcal{E}^{2}-c^{2} \mathcal{B}^{2}\right) \delta_{i j}+7 c^{2}\left(\mathcal{B}_{i} \mathcal{B}_{j}\right)\right] \\
\mu_{i j} \approx \delta_{i j}+\frac{4 \lambda_{e}^{3} \alpha^{2}}{45 m_{e} c^{2}}\left[2\left(c^{2} \mathcal{B}^{2}-\mathcal{E}^{2}\right) \delta_{i j}+7\left(\mathcal{E}_{i} \mathcal{E}_{j}\right)\right]
\end{array}
$$

In the following, the restriction to the special case of the vacuum perturbed by a magnetic field and a linearly polarised light probe is considered. In that case, the first order correction to the velocity of the light can be expressed as [7]:

$$
v / c \approx 1-a \mathcal{B}^{2} \sin ^{2} \theta_{B}\left(\frac{\epsilon_{0} \lambda_{e}^{3} \alpha^{2}}{45 m_{e}}\right)
$$

where $\theta_{B}$ is the angle between the direction of the photon propagation and the $\mathcal{B}$ field and $a$ is constant either equal to 8 or 14 for photons polarised perpendicular and parallel to $\mathcal{B}$ respectively. The quantum vacuum behaves as a birefringent medium when submitted to a magnetic field (this induced birefringence effect has been known for material optical science since 1905 as the Cotton-Mouton effect [8]). When the light beam propagates a right angle with respect to magnetic field, the refractive index difference between light polarised parallel and perpendicular to $\mathcal{B}$ is given by:

$$
\Delta n \approx\left(\frac{6 \epsilon_{0} \lambda_{e}^{3} \alpha^{2}}{45 m_{e}}\right) \mathcal{B}^{2}
$$


and corresponds for example to a value $\Delta n \approx 3.6 \cdot 10^{-22}$ in $9.5 T$ field, which is really challenging to detect. A possibility to observe the photon-photon interaction due to the $Q E D$ vacuum is to measure the ellipticity $\psi_{Q E D}$ acquired by a laser light beam, initially linearly polarised, after its passage trough the vacuum submitted to a transverse magnetic field [4]. This ellipticity is equal to:

$$
\psi_{Q E D}=\pi \Delta n\left(\frac{l}{\lambda}\right) \sin (2 \theta)
$$

where $l$ is the length of the optical path in the magnetic field region, $\lambda$ is the light wavelength and $\theta$ is the angle between the light electrical vector and the applied field $\mathcal{B}$. An important point is that the ellipticity is cumulative upon reflection so the effect can be accumulated by making the light travel back and forth in the magnetic field. To fix the ideas, the maximum of ellipticity acquired by a laser light beam with $\lambda=1.55 \mu \mathrm{m}$ propagating in a $9.5 \mathrm{~T}$ field over a length $l=2500 \mathrm{~m}$ is equal to $2 \cdot 10^{-12} \mathrm{rad}$.

\subsection{Beyond the pure QED prediction}

The EHW effective Lagrangian density can be further extended to include contributions of hypothetical neutral light spin zero particles that couple to two photons such as axions [9], [10]. In that case, the initially linearly polarised light beam is expected to acquire a small apparent rotation $\Theta$ due to a linear dichroism and a small ellipticity $\Psi$ [5]. Both effects can be expressed in natural Heaviside-Lorentz units where $1 T=195(\mathrm{eV})^{2}$ and $1 \mathrm{~m}=5 \cdot 10^{6} \mathrm{eV}-1$ by:

$$
\begin{aligned}
& \Theta \approx\left(\frac{\mathcal{B}^{2} l^{2}}{16 M^{2}}\right) \sin (2 \theta) \\
& \Psi \approx\left(\frac{\mathcal{B}^{2} l^{3} m^{2}}{96 \omega M^{2}}\right) \sin (2 \theta)
\end{aligned}
$$

where $M$ is the inverse coupling constant, $m$ is the hypothetical particle mass and $\omega$ is the photon energy.

The axions are the consequence of the absence of CP violation in QCD (Quantum Chromodynamics) [9], [10]. The interest in such hypothetical neutral light spin zero particles lies beyond particle physics since they are considered as good dark matter candidates for the universe [11]. The development of a pure laboratory experiment for the production and the detection of possible dark matter candidates offers the opportunity to perform a study independent of astrophysical and cosmological assumptions.

\section{Proposed Experiment}

\subsection{Principle}

To measure the VMB expressed by (6) and (8), the experimental approach must focus on two main requirements. First, the ellipticicity acquired by the initially 
linear polarised laser beam propagating through the vacuum submitted to a transverse $\mathcal{B}$ field must be boosted by using an optical cavity. Second, the ellipsometer to measure the extremely small polarisation change must be optimised together with the optical detection technique. As this will be presented, the main limiting factor of this experiment is the shot noise of the photodetector and the optical detection must succeed to overcome this limitation.

\section{$3.2 \quad$ Existing set-up}

An ideal implementation for the optical cavity is inside a long superconducting dipole magnet such as the ones developed and manufactured for the LHC (Large Hadron Collider) under construction at CERN. Full scale prototypes have been built to validate the magnet design and will no longer be used for the accelerator. One of the first generation was already decommissioned and is at present in routine operation in the CAST experiment to detect solar axions [12]. Third generation twin aperture superconducting LHC dipole prototypes offer the advantage to have a magnetic field region of length equal to $14.3 \mathrm{~m}$ [13]. The maximum field they can reach is around $9.6 \mathrm{~T}$ at $1.9 \mathrm{~K}$ which corresponds to the limit of superconducting cables in the peak field region. They have been extensively tested and measured magnetically in the SM18 facility at CERN [14] which provides the cryogenic environment for cooling large superconducting magnets below the lambda point of liquid He. To measure the ellipticity expressed by (6) and (8), a single one of this magnet has $\mathcal{B}^{2} l \approx 1290 T^{2} m$ and $\mathcal{B}^{2} l^{3} \approx 263 \cdot 10^{3} T^{2} m^{3}$ which are respectively about 10 and 20 times larger than the magnetic characteristics of the VMB experimental setup providing the present reference results [15]. Additional gains can be obtained by optimising the performance of the optical cavity, of the magnet power supply and of the detection principle as this will be presented in a next paragraph. The field measurements of these superconducting dipoles were performed with rotating coils kept at room temperature by sliding warm fingers inside the apertures called anticryostats [16]. They have a $40 \mathrm{~mm}$ aperture and a length of $19.6 \mathrm{~m}$ to go through the Cryogenic Feed Box (CFB) and have been used opened at both ends or closed and pumped down. Fig.1 shows a general layout of a superconducting dipole on a test bench connected to its CFB and fitted with anticryostats.

\subsection{Technical solutions and integration}

To obtain a light path inside the magnetic field region as long as possible, an optical cavity must be used. Cameron et al. [15] developed a multipass cavity made of two high reflectivity spherical mirrors with one having a hole in the centre to allow the light to enter and to exit. With such type of cavity, up to about 600 reflections was obtained. Because of constraints mainly coming from the small diameter of the LHC dipole apertures, a better choice is to consider a Fabry-Perot cavity. As a consequence, a Pound-Drever scheme must be used to lock the laser frequency with the resonant frequency of the cavity.

In order to operate the optical cavity at room temperature, the anticryosats will 
be used as a part of the vacuum chamber with endcaps at both extremities. The mirrors of the cavity will be integrated inside endcaps well outside the magnetic field to avoid spurious effects. The tuning of the orientation of the mirrors will be done using piezoelectric actuators. Preliminary studies of the effect of the ground motions on the mirror orientation in our experimental hall at SM18 have been performed and angular stability better than $3 \mu \mathrm{rad}$ was measured [17], giving a present upper limit of $\sim 100$ for the finesse $F$ of the optical cavity. An increase of one order of magnitude, necessary to reach $F \sim 1000$, is expected with future improvements of both opto-mechanical design and piezoelectric actuators control.

\subsection{Optical Detection Technique}

The layout of the experiment with the optical detection part is given in Fig.2. Using a half-wave plate mounted in a high-speed rotation stage, the linear polarization state of the laser beam can be modulated in the $\mathrm{kHz}$ range. After the second pass through the half-wave plate, the laser beam will retrieve to the first order, its linear polarization state with the small VMB rotation angle induced by the vacuum submitted to the transverse $\mathcal{B}$ field inside the optical cavity. Then a quarter-wave plate will convert the quasi-linear polarized laser beam coming out from the cavity into a quasi-circular one. Finally, a polarizer will ensure a linear and optimal conversion of the induced ellipticity into a power modulation of the laser beam. A photodiode will be used for the detection.

The modulation of the magnetic field has two main drawbacks and should be avoid. First it decreases the VMB signals by reducing the value of the effective magnetic field producing the effect and second it imposes detection at low frequency due to the voltage limitation of the magnet power supply. On the contrary, a modulation of the laser beam polarisation can be made in the $\mathrm{kHz}$ range, thus dramatically reducing detrimental effects of acoustic vibrations and temperature fluctuations. Moreover, as two of the three main noise sources encountered in birefringence measurement experiments [18], namely the Relative Intensity Noise (RIN) of the laser source and the noise of the detection, are frequency dependent as they exhibit $1 / f$ noise at low frequency, it is crucial to work above the $1 / f$ noise cut-off frequencies.

\section{Expected performance}

Table 1 gives a summary of the different noise sources that contribute to the limitations of the experimental sensitivity for the measurement of extremely small birefringence. Considering only the first three noise sources as the main ones, the following expression for the relative noise power can be obtained:

$$
\tilde{P}_{\text {noise }} \equiv \frac{P_{\text {noise }}}{P}=\left(R I N+\frac{2 e}{i_{p h}}+\frac{4 k T_{\text {eff }}}{R_{L} i_{p h}^{2}}\right) \Delta f
$$

$k$ is the Boltzmann constant, $i_{p h}$ is the photodiode average current, $R_{L}$ represents the load resistance of the photodiode and $T_{e f f}>T_{a m b}$ and $\Delta f$ are the effective 
temperature and bandwidth of the detection system respectively. Table 1 has been built using typical maximum ratings of photodiode characteristics and the best laser diode in terms of RIN. Above a few $\mathrm{kHz}$, Table 1 shows that the relative noise power coming from the three main noise sources are very close, even if the shot noise gives the main contribution. For an acquisition time of a few seconds $(\Delta f \sim 1 H z)$, the relative variation of the electrical power delivered by the photodiode should be at least $-150 \mathrm{~dB}$ to distinguish the effect of the VMB from the noise. This implies a modulation depth of at least $3 \cdot 10^{-8}$ for the photodiode current $i_{p h}$, i.e. a minimal ellipticity of the same order of magnitude. $\psi_{Q E D}$ given in (6) is achievable considering $\Delta f \sim 1 \mathrm{mHz}$ (acquisition time of a few tens of minutes) and a finesse $F \approx 1000$ for the optical cavity, these two values being realistic. One order of magnitude can be probably gained via a simultaneous increase of 10 of the acquisition time (to reach few hours) and of the optical power impinging on the photodiode $(10 \mathrm{~mW})$. However, long time acquisitions bring a lot of drawbacks due to long term drifts of electronics and temperature.

To gain several additional orders of magnitude in the measurement sensitivity necessary to study the VMB down to a level much below the pure QED prediction, a breakthrough must be done: the shot noise limit must be overcome. The shot noise constitutes to a strong limitation because the VMB induces only a hugely slight modulation of the optical power. Thus, the suppression of the constant part of the optical power would solve the problem. In electronics for example, a direct current is suppressed by inserting a capacitance in the circuit. Based on this analogy, a novel optical detection scheme is under study [19]. It consists in the transposition of the modulated laser beam to a frequency far away from the optical carrier frequency. With optical filtering, the constant part of the optical power would be partially suppressed and this will dramatically increase the modulation depth of the laser beam impinging on the photodiode. Consequently, both RIN and shot noise, that were strongly limiting the measured modulation depth, will get a minor influence in comparison with the noise of the detection (Johnson noise in the load resistance and amplification noise). By using a cooled photodiode with highly optimised electronics, a gain of at least two orders of magnitude is expected, all the other parameters $(F, \Delta f)$ being unchanged.

\section{Conclusion and future developments}

The reuse of unique equipments developed at CERN for the LHC project offers a rare opportunity to build a "low cost" experiment which can provide the values or some new limit values of the mass and of the coupling constant to two photons of possible dark matter candidates such as axions. In the most conservative view concerning the optical detection technique, the measurement of the VMB can be improved by at least 2 orders of magnitude with respect to the present reference results [15].

To complement and cross check the VMB measurements, a second experiment is also currently considered. Its aim is to detect the photon regeneration from axions 
using the concept of "a shining light through the walls" [20]. The realisation of this additional setup is also feasible with a minimum of investment. It is planned to integrate it in the second aperture of the 15-meters long LHC prototype superconducting magnet with an optimisation of the photon-to-axion and axion-to-photon converters [21].

\section{References}

[1] W. Heisenberg and H. Euler : Z. Phys. 98 (1936) 714.

[2] V. S. Weisskopf : Mat.Fys. Medd. Dan Vidensk. Selsk. 14 (1936) 1.

[3] J. Schwinger : Phys. Rev. 82 (1951) 664.

[4] I. Iacopini and E. Zavattini: Phys. Lett. 85B (1979) 151.

[5] L. Maiani, R. Petronzio, and E. Zavattini: Phys. Lett. 175B (1986) 359.

[6] S. G. Mamaev, V. M. Mostepanenko, and M. I. Eides: Sov. J. Nucl. Phys. 33 (1981) 569.

[7] S. L. Adler: Ann. Phys. 67 (1971) 599.

[8] A. Cotton and H. Mouton: Compt. Rend. Acad. Sci. (Paris) 141 (1905) 317.

[9] S. Weinberg: Phys. Rev. Lett. 40 (1978) 223.

[10] F. Wilczek: Phys. Rev. Lett. 40 (1978) 279.

[11] M. S. Turner: Phys. Rep. 197 (1990) 67.

[12] K. Zioutas et al.: Nucl. Inst. and Meth. A 425 (1999) 480.

[13] The LHC Study Group: CERN/AC/95-05 (1995) 84.

[14] L. Bottura et al.: IEEE Trans. Appl. Superconduct. 12 (2002) 211.

[15] R. Cameron et al.: Phys. Rev. D 47 (1993) 3707.

[16] O. Dunkel, P. Legrand, and P. Sievers: AIP Conferences proceedings 710(1) (2004) 494.

[17] P. Schnizer and N. Smirnov CERN/LHC/MTA Memorandum, May 8, (2001).

[18] L. Duvillaret et al.: J. Opt. Soc. Am. B 19 (2002) 2692.

[19] L. Duvillaret and P. Pugnat: to be submitted to Europhys. Lett.

[20] K. Van Bibber et al. Phys. Rev. Lett. 59 (1987) 759.

[21] L. Duvillaret, M. Finger, M. Kral, P. Pugnat, A. Siemko, J. Zicha: to be submitted to Nucl. Inst. and Meth. A. 
Table 1. Overview of the various sources of noise impacting the measurement of the VMB.

\begin{tabular}{|c|c|}
\hline Noise source & Principal characteristics \\
\hline Laser RIN & Relative noise power \\
\hline $\begin{array}{l}\text { Photodiode } \\
\text { shot noise }\end{array}$ & $>-155 \mathrm{~dB} / \mathrm{Hz} \overbrace{}^{\text {Relative noise power }} \begin{array}{l}\text { Optical power } \sim 1 \mathrm{~mW} \\
\text { Optical response } \sim 1 \mathrm{~A} / \mathrm{W}\end{array}$ \\
\hline Electronic unit noise & $\begin{array}{l}10 \mathrm{k} \Omega \text { transimpedance amplifier } \\
\text { \& spectrum analyzer }\end{array}$ \\
\hline Acoustic vibrations & Especially detrimental below $\sim \mathrm{kHz}$ \\
\hline $\begin{array}{l}\text { Temperature } \\
\text { fluctuations }\end{array}$ & Especially detrimental below $\sim \mathrm{Hz}$ \\
\hline Intrinsic noise & From active optical elements if any. \\
\hline $\begin{array}{c}\text { Fluctuations of laser } \\
\text { polarization state }\end{array}$ & $\begin{array}{l}\text { Of fiist importance only if the measurement of the vacuum magnetic birefringence is } \\
\text { based on the magnetic field induced variation of the laser polarization. Possible } \\
\text { suppression by use of high grade polarizer(s) with the drawback of converting } \\
\text { polarization fluct uations into intensity fluctuations. }\end{array}$ \\
\hline
\end{tabular}




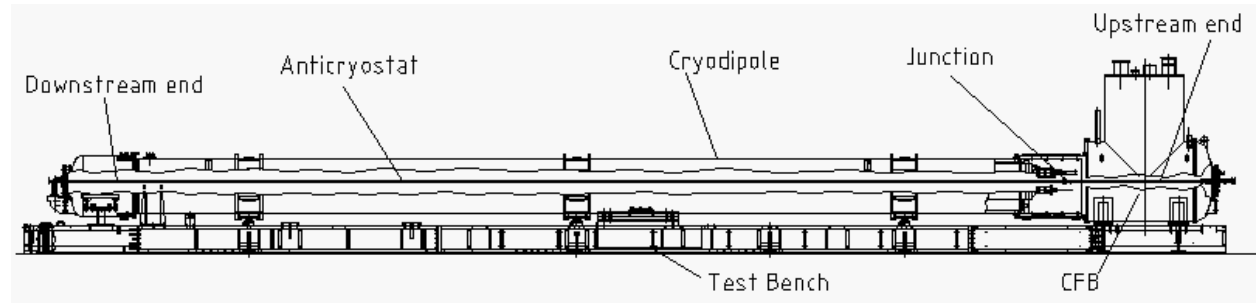

Fig. 1. Layout of a cold test bench for dipole magnets equipped with anticryostats and connected to its Cryogenic Feed Box (CFB).

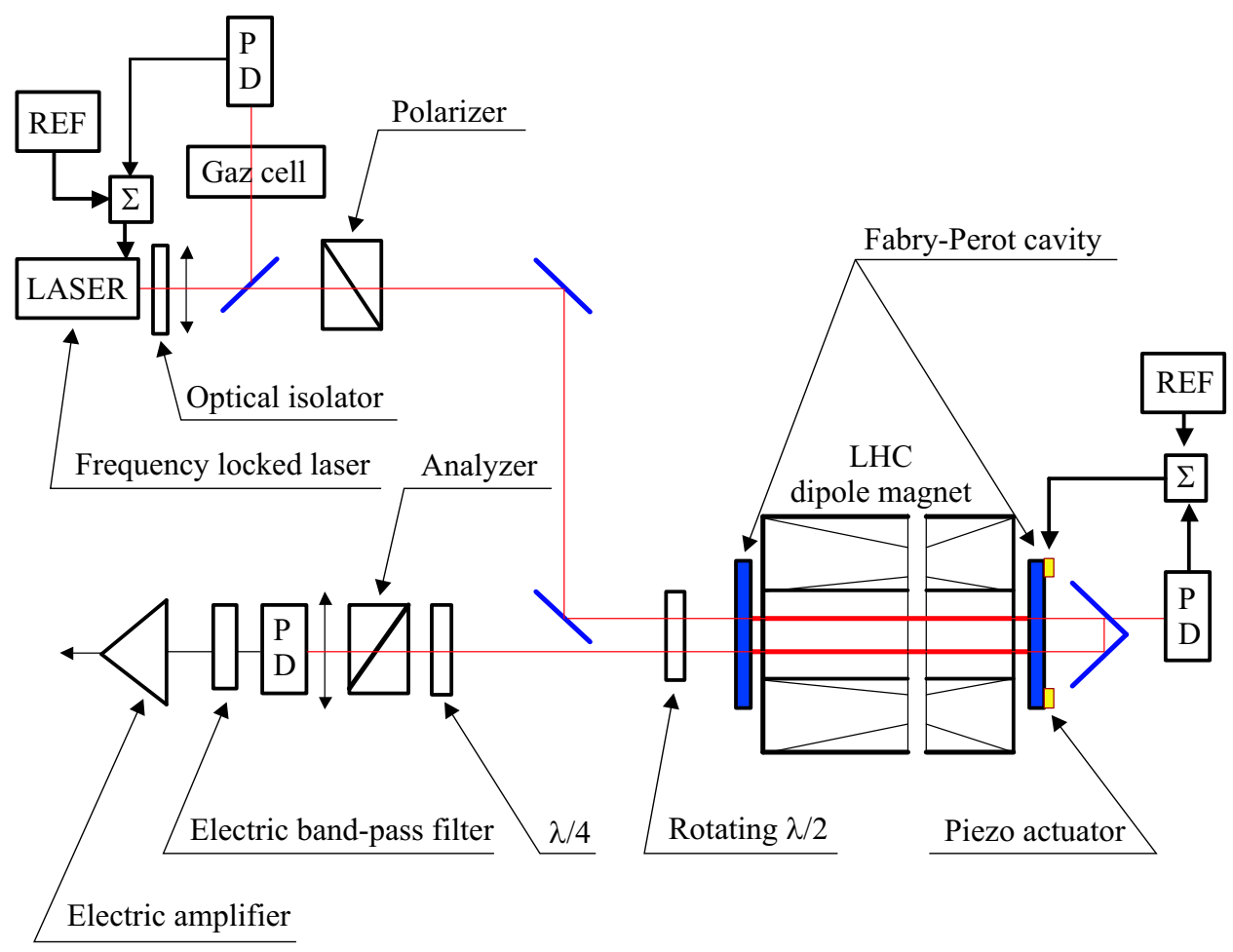

Fig. 2. Layout of the optical detection part of the experiment to measure the VMB. 